\title{
Power Thresholds and Pump Depletion in Brillouin Fiber Amplifiers
}

\author{
Fabien Ravet ${ }^{\S}$, Jeffrey Snoddy, Xiaoyi Bao* and Liang Chen
}

Department of Physics, University of Ottawa, Canada

\begin{abstract}
The Brillouin fiber amplifier (BFA) has been used in Brillouin optical time (frequency) domain analyzer based sensors. The performance of this sensor depends on pump depletion which occurs when the threshold is reached and when the Brillouin gain is saturated. Although threshold definitions are common for Brillouin fiber generators (BFG), there is no explicit threshold definition for BFA. More importantly we find the maximum gain point corresponds to the optimized distributed sensing system where the depletion effect remains small to moderate. We find that only the method based on the determination of the power for which the pump becomes depleted, and hence, the Stokes gain saturated (i.e. inflexion method), remains valid for BFA. We also derive an analytical relation accounting for the various sensor key parameters such as fiber type and length as well as input Stokes power. The thresholds obtained with the analytical relation are within $10 \%$ of experimental and numerical values, which is maximum power that can be launched for a given optimum sensing configuration.
\end{abstract}

\section{INTRODUCTION}

Since the first demonstration of light amplification in optical fibers with stimulated Brillouin scattering (SBS), the Brillouin fiber amplifier (BFA) configuration [1] has been implemented in a wide range of applications. From tunable narrowband filtering [2] to the control of pulse propagation in optical fibers $[3,4]$, the interest for SBS never ceased. Among all these applications, we want to mention the works of Horiguchi and Culverhouse, both in 1989, where the authors demonstrated that the BFA can be used to measure strain and temperature respectively, initiating a prolific research in fiber optic sensors [5,6]. Associated with frequency or time domain modulation of the pump (or Stokes) beam, those physical quantities can be measured along the whole fiber length, leading to the development of distributed Brillouin sensors (DBS).

Recently, we have shown that pump depletion influences the DBS performance [7]. More specifically, the sensor parameters, such as spatial resolution, Brillouin frequency resolution and sensing length, depend on pump and Stokes power levels as well as on the sensing fiber properties. On the one hand, a large Stokes input power enhances electrostriction, reinforcing the interaction with the pump, which is at the origin of pump depletion. That also improves spatial, strain and temperature resolutions. On the other hand, the sensor must be able to measure strain and temperature over its entire length with uniform resolution, which is impossible when pump depletion is strong, as the Brillouin spectrum will be distorted leading to the temperature or strain measurement error. There is then a limit to the power that can be fed into the fiber in order to achieve optimum sensing

*Address correspondence to this author at the Department of Physics, University of Ottawa, 150 Louis-Pasteur, Ottawa, Ontario, Canada, K1N 6N5; Tel: (613) 562-5800, Ext. 6911; Fax: (613) 562-5190;

E-mail:xbao@uottawa.ca

${ }^{\S}$ Fabien Ravet is currently with Omnisens, Switzerland

(fabien.ravet@omnisens.ch) operation, i.e. in order to achieve a frequency resolution in the $\mathrm{MHz}$ range and a spatial resolution in the meter range over sensing lengths of tens of kilometers. As a consequence, the need for a criterion that determines how much depletion can be tolerated without affecting significantly the sensor performance is certainly critical. We recall Smith's threshold [8] expressed as

$$
P_{B F G} \approx 21 A_{\text {eff }} / \chi_{S B S} g_{B} L_{e f f} \equiv 21 P_{e q} \text {, }
$$

where $g_{B}$ is the Brillouin gain coefficient, $\chi_{S B S}$ is the depolarization factor, which was inserted in Eq. 1 to account for the randomization of the polarization state in the fiber [9], $A_{\text {eff }}$ and $L_{\text {eff }}=\left(1-e^{-\alpha L}\right) / \alpha$ are the fiber effective area and length, respectively. $L_{\text {eff }}$ is function of fiber attenuation $(\alpha)$ and length $(L)$. We define $P_{e q}=A_{\text {eff }} \chi_{S B S} g_{B} L_{e f f}$ as the equivalent to the input Stokes power that would give an amplification factor of 21. Note that with modern optical fibers the factor 21 is replaced by 19 [10]. Unfortunately, it only applies to the Brillouin fiber generator (BFG). This threshold did not consider the input Stokes power level, which is a key parameter of the BFA. To the best of our knowledge, there is no such definition applying to the BFA configuration and that account for depletion effects in the DBS, especially for long sensing lengths (tens of kilometers). Such a power threshold definition would then set an upper limit to the input powers, guaranteeing an optimum operation of the sensor.

The purpose of this letter is to introduce a power threshold $\left(P_{t h}\right)$ definition for the BFA configuration which sets an upper limit to the distributed Brillouin sensor based on Brillouin gain or loss. We first discuss threshold definitions that are commonly used in the BFG. We show that only the definition based the fact that the pump starts to be depleted once the threshold is reached, can be applied to the BFA configuration [11]. We then derive an analytical expression for the threshold in Brillouin fiber amplifier. We carried out experimental and numerical characterization of BFG and BFA configurations to verify our new threshold definition. The numerical study uses the same approach as that of reference 
(a)
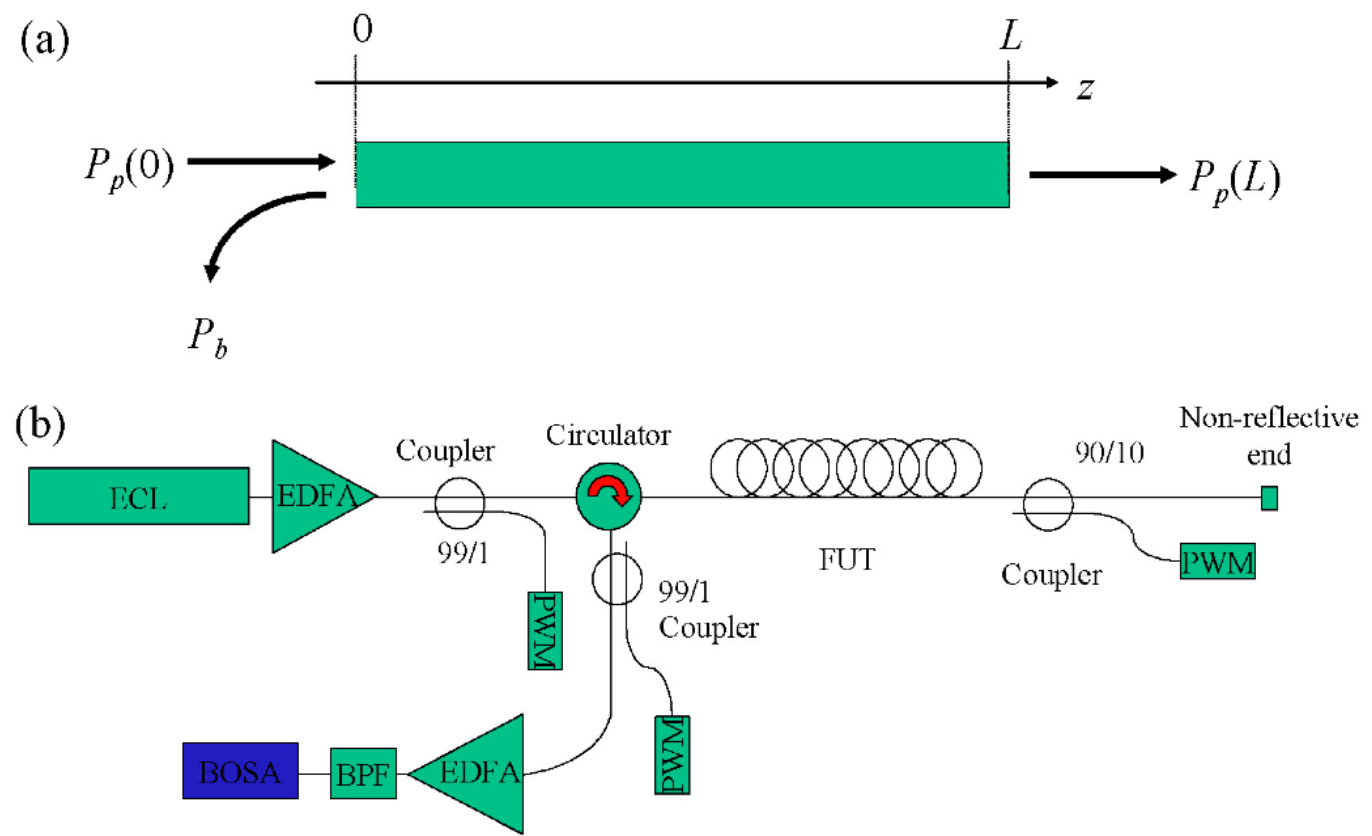

Fig. (1). (a) Schematic of the Brillouin generator configuration. (b) Experimental set-up; EDFA: Erbium Doped Fibre Amplifier; PWM: Power Meter; BPF: Band Pass Filter, BOSA: Brillouin Optical Spectrum Analyzer; FUT: Fibre Under Test.

7. We solved the coupled intensity equations according to the Newton-Raphson method. We also added a term describing the effect of Rayleigh backscattering [12]. In all simulations the following parameter values are used: $\chi_{S B S}=2 / 3, g_{B}=$ $2.15 \times 10^{-11} \mathrm{~m} / \mathrm{W}, A_{\text {eff }}=83.7 \mu \mathrm{m}^{2}$.

\section{BRILLOUIN FIBER GENERATOR}

As illustrated in Fig. (1), the Brillouin fiber generator (BFG) is composed of a pump signal $\left(P_{p}(0)\right)$ and an optical fiber. Backward lightwave is emitted by Rayleigh and spontaneous Brillouin scattering $\left(P_{b}\right) . P_{b}$ is routed through an optical circulator before being measured by a power meter. Fig. (1b) shows in details the experimental set-up implemented for the backscattering measurements. In order to discriminate the respective influence of the scattering components, we added a Brillouin Optical Spectrum Analyzer (BOSA) which has a frequency resolution of $10 \mathrm{MHz}$ [14]. We used a $1554 \mathrm{~nm}$ external cavity laser whose linewidth is less than $100 \mathrm{kHz}$ and which is boosted by a $21 \mathrm{dBm}$ erbium doped fiber amplifier. The fibers are ITU G.652 types [13] of 2 and $20 \mathrm{~km}$. An example of backscattered power versus input pump is presented in Fig. (2) for a $20 \mathrm{~km}$ long stepindex fiber. Fig. (2a) represents the measurement of the total backscattered power, which is the sum of Rayleigh $\left(P_{R}\right)$ and Brillouin $\left(P_{s}\right)$ scatterings. Fig. (2b) shows the comparison of the measurements with the results of numerical simulations. Well below the $1 \%$ input power line $\left(P_{p}(0)-20 \mathrm{~dB}\right.$, both numerical and experimental curves show that Rayleigh scattering dominates the process. At the contrary, in the neighborhood and above the $1 \%$ input power line, Brillouin scattering is the major contribution to the backscattering signal.

As experimental threshold definition, we use the input power at which $P_{b}$ reaches $1 \%$ of the input pump power $(1 \%$ criterion) $[10]$. From the preceding paragraph, we can conclude that near threshold Rayleigh scattering is negligible. It is then not an issue to apply the $1 \%$ criterion either to $P_{b}$ or
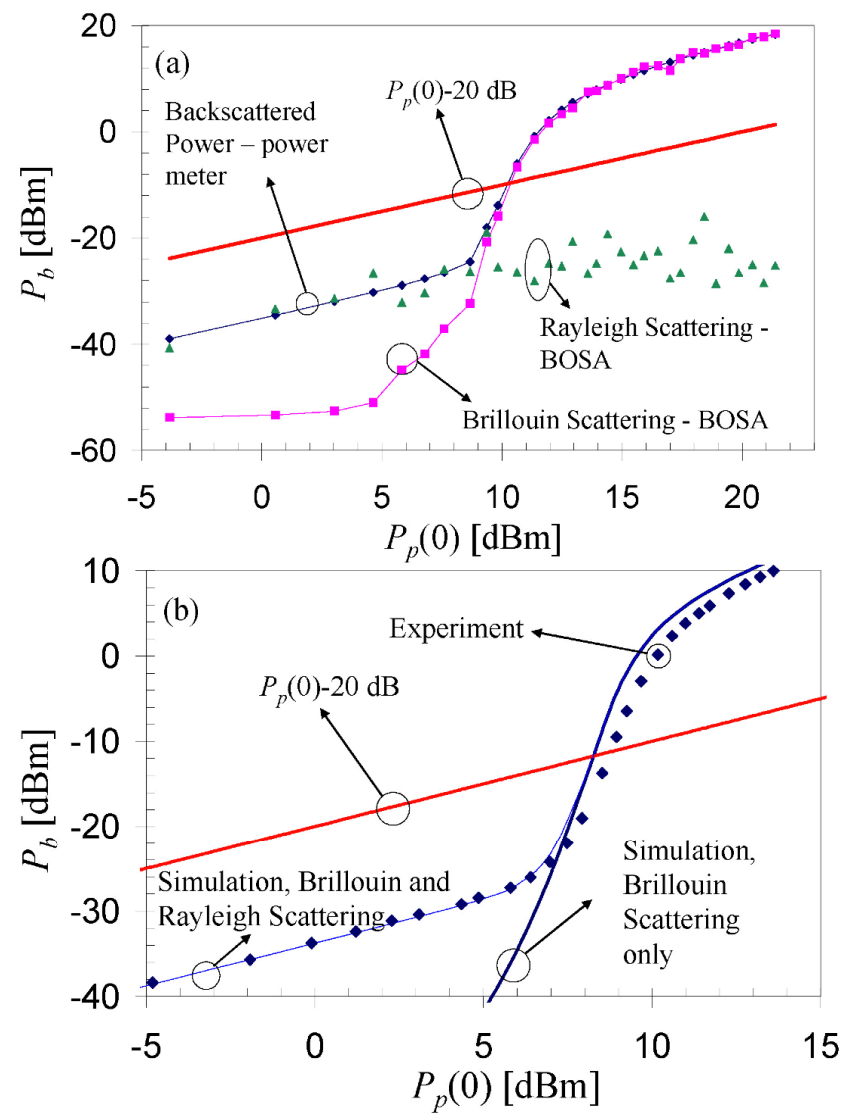

Fig. (2). Results of numerical simulation (lines) and measurement (full diamond) of backscattered power.

to $P_{s}$. It is illustrated by the intersection of the plain and the dashed curves in Figs. (2,3). In Fig. (3), we have also drawn the derivative of Stokes backscattered power versus input pump $\left(d P_{s} / d P_{p}\right)$, which shows how the stimulated regime evolves with pump power. The rate of increase of the $P_{s}$ against $P_{p}(0)$ first rises, which is a signature of the stimulated 
regime. Then the rate decreases because pump depletion is not negligible anymore. Interestingly, we observe that the intersection of $P_{s}$ curve with the $1 \%$ criterion line always occurs at the maximum of $d P_{s} / d P_{p}$. That intersection corresponds to the inflexion point of the $P_{s}$ curve. The inflexion point can then be considered as an alternative definition of the threshold based on the $1 \%$ criterion. It gives the input power for which the pump power starts to be depleted [11]. Note that threshold determination by the $1 \%$ criterion only requires the use of power meters to measure input and backscattered powers as Fig. (2) clearly shows that Rayleigh backscattering can be neglected. The inflexion method can be used similarly with the total backscattered power $P_{b}$.

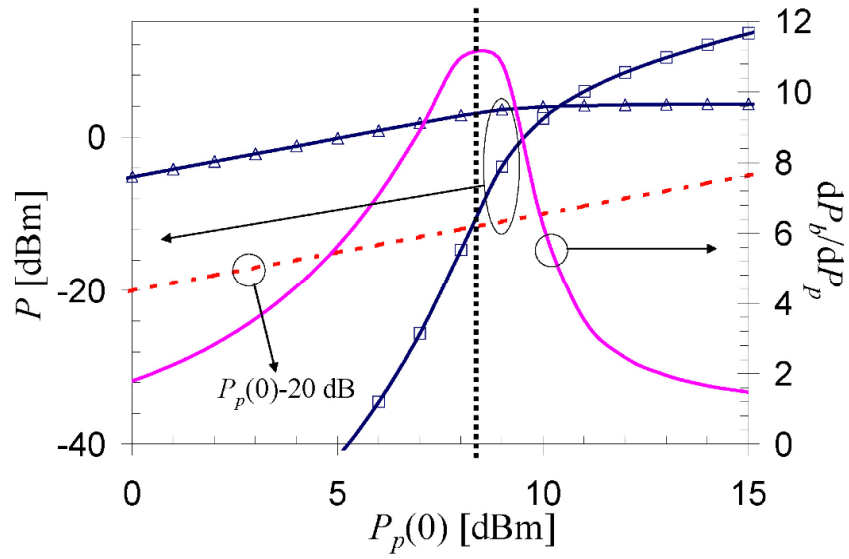

Fig. (3). Stokes backscattered $P_{s}$ (squares) and transmitted $P_{p}(L)$ (triangles) powers as well as the rate of change of the backscattered power against input pump power $d P_{s} / d P_{p}$.

As illustrated in Fig. (4), $P_{t h}$ depends on the fiber length. Based on Smith's definition, the threshold derived from measurements with the $1 \%$ criterion and the inflexion method as well as the threshold calculated from the simulations of the coupled intensity equations [7] show similar trends. Note that we assumed in this model that $P_{b}$ is initiated from a localized Stokes source placed at $z=L[15]$, which corresponds to an equivalent input Stokes power of $411 \mathrm{nW}$. This value is obtained by assuming spontaneous regime; the Stokes power is proportional to the number of acoustic phonons. At room temperature, the scattered power is given by $k_{B} T v_{\mathrm{s}} \Delta v_{B} / v_{B}$, where $v_{s}$ is the Stokes frequency, $v_{B}$ is the Brillouin frequency $\left(v_{B} \approx 11 \mathrm{GHz}\right), \Delta v_{B}$ is the natural linewidth of the Brillouin spectrum $\left(\Delta v_{B} \approx 32 \mathrm{MHz}\right), k_{B}$ is the Boltzman constant and $T$ is the temperature. Smith's definition is systematically higher than both experimental and simulation results. We believe that there are two reasons why Smith's and the other definitions do not match. First, Smith's definition is derived with an undepleted approximation, valid for $P_{p}(0)<<P_{\text {Smith }}$. Second, the $1 \%$ criterion has no physical relationship with Smith's definition as it is defined as the input pump power "for which the backward stimulated Stokes power equals input pump power at $z=0$ ". Examining Fig. (3), it is obvious that such definition is not practical: the backscattered power is much lower than the input power due to pump depletion. These are the reasons why the $1 \%$ criterion and the inflexion method are always preferred for experimental determination of the threshold.

\section{BRILLOUIN FIBER AMPLIFIER}

The BFA configuration [1] requires that a Stokes wave is injected at the opposite $\left(P_{s}(L)\right)$ end of the fiber as illustrated in Fig. (5). The two counter-propagating lightwaves are coupled through electrostriction, which leads to the amplification of the Stokes at the expense of the pump, inducing higher pump depletion. In our experimental set-up, we measured the output Stokes wave $\left(P_{s}(0)\right)$ at the pump input. Stokes and pump sources are Erbium doped fiber lasers, whose linewidths are smaller than $100 \mathrm{kHz}$. Their frequency difference is controlled with a phase locked loop and kept to $v_{B}$ of the fiber under test. Optical circulators are used to route the input and output signals at both ends of the fiber. The signals are detected using power meters.

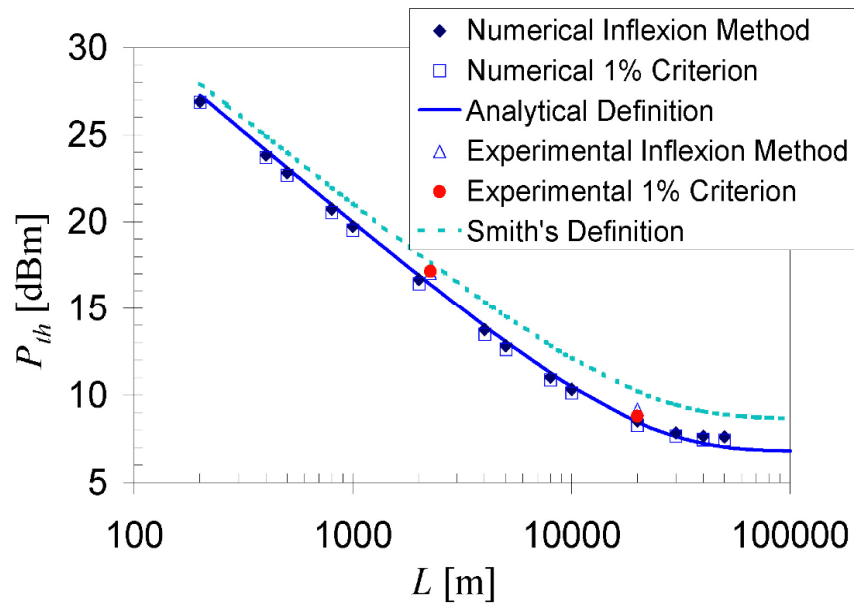

Fig. (4). $P_{t h}$ vs $L$ (Smith's and analytical definitions refer to Eqs. 1 and 6 , respectively).

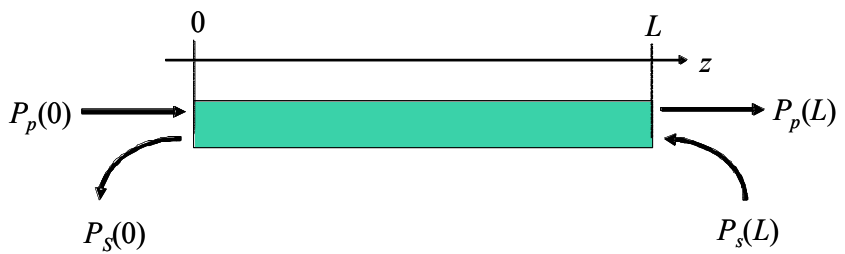

Fig. (5). Schematic of the Brillouin amplifier configuration.

We draw $P_{s}(0)$ vs $P_{p}(0)$ as represented in Fig. (6) for a BFA made of a step-index fiber with $L=10 \mathrm{~km}$. We see that $P_{s}(L)=1 \mu \mathrm{W}$ and $P_{s}(L)=10 \mu \mathrm{W}$ belong to the same regime. From Fig. (6), it is clear that Stokes curves are shifted to the upper left corner of the graphic as $P_{s}(L)$ increase. This makes the use of the $1 \%$ criterion unoperational for the determination of the threshold in a Brillouin amplifier. In the case of $P_{s}(L)=1 \mu \mathrm{W}$, there are two intersections between the $1 \%$ line and $P_{s}(0)$ curve. In the other case, there is no intersection at all. Then we verify that the inflexion method as an experimental threshold definition is still valid. $P_{t h}$ obtained from both experimental $\left(P_{\exp }\right)$ and numerical $\left(P_{\text {num }}\right)$ results of Fig. (6) are presented in Table $\mathbf{1 .}$

As mentioned in the introduction, an analytical definition is certainly important for distributed sensing applications. In order to derive a simple relation, we consider the same argument as Smith [8] which is that the threshold is the input pump such that the output Stokes is equal to the input pump. That criterion, applied to the Smith's approximation [8], leads to the following equality

$f(x)=x-I_{s}(L) \exp \left(\chi_{S B S} g_{B} x L_{e f f}\right)$ 
where $I_{s}(L)$ is the input Stokes intensity. The root of that equation is the threshold intensity, which can be obtained numerically with the Newton-Raphson method. This method is based on a Taylor expansion of $f$ near its zero:

$$
f(x+\delta) \approx f(x)+f^{\prime}(x) \delta+f^{\prime \prime}(x) \delta^{2} / 2+\ldots
$$

When $|\delta|<<1$, which happens when $f(x+\delta)$ very close to zero, higher order terms can be neglected. We can then write that

$$
|f(x) / \delta| \approx\left|f^{\prime}(x)\right|<\eta, \quad 0<\eta<+\infty .
$$

The condition on $\eta$ is imposed by the fact that the first and second order derivatives of $f$ have to be defined. Calculating explicitly $f$ ' and using the argument that $\eta$ is a finite number, we find that the power threshold is bounded as follows

$\left.\left.P_{e q} \ln (1-\eta) P_{e q} / P_{s}(L)\right\}<P_{B F A}<P_{e q} \ln (1+\eta) P_{e q} / P_{s}(L)\right\}$

$P_{e q}$ is defined by Eq. 1. Additionally, Eq. 5 imposes that $\eta<1$. We can then express the threshold as

$P_{B F A}=P_{e q} \ln \left\{\zeta P_{e q} / P_{s}(L)\right\} \quad 1-\eta<\zeta<1+\eta$.

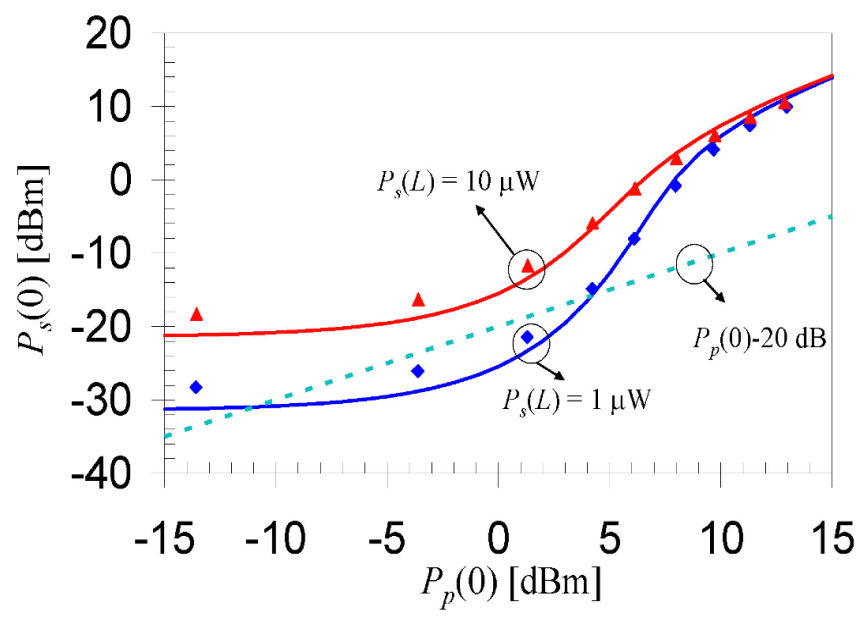

Fig. (6). Experimental (symbols) and numerical (lines) $P_{s}(0) v s$ $P_{p}(0)$ for $P_{s}(L)=1 \mu \mathrm{W}$ and $P_{s}(L)=10 \mu \mathrm{W}$.

Table 1. $P_{t h}$ of the $10 \mathrm{~km}$ Long BFA

\begin{tabular}{|c|c|c|c|c|}
\hline $\mathbf{P}_{\mathbf{s}}(\mathbf{L})[\mathbf{d B m}]$ & $\mathbf{L}_{\text {eff }}[\mathbf{m}]$ & $\mathbf{P}_{\text {exp }}[\mathbf{d B m}]$ & $\mathbf{P}_{\text {num }}[\mathbf{d B m}]$ & $\mathbf{P}_{\text {BFA }}[\mathbf{d B m}]$ \\
\hline \hline-30 & 8073.1 & 6.2 & 6.8 & 6.7 \\
\hline-20 & 8101.9 & 5.0 & 5.5 & 4.7 \\
\hline
\end{tabular}

We found that $\zeta=0.97$ by fitting Eq. 6 to the data of Fig. (4) (the best fit is the curve labeled as analytical definition in Fig. (4)). The root-mean-square deviation of the relative error is better than 3\%. The threshold calculated with Eq. 6 for the $10 \mathrm{~km}$ long step-index fiber is reported in Table 1. The analytical threshold $\left(P_{B F A}\right)$ is within $10 \%$ of the experimental and numerical values. Fig. (7) illustrates that analytical and simulation results agree well, except at higher $P_{s}(L)$ because pump depletion is not accounted for in Eq. 6 derivation. However, in distributed sensing, large depletion has to be avoided as it degrades the sensor performance. $P_{B F A}$ is then a threshold definition that gives an upper limit to the pump input power for a given optimum sensing configuration i.e. for given input Stokes power and fiber characteristics.
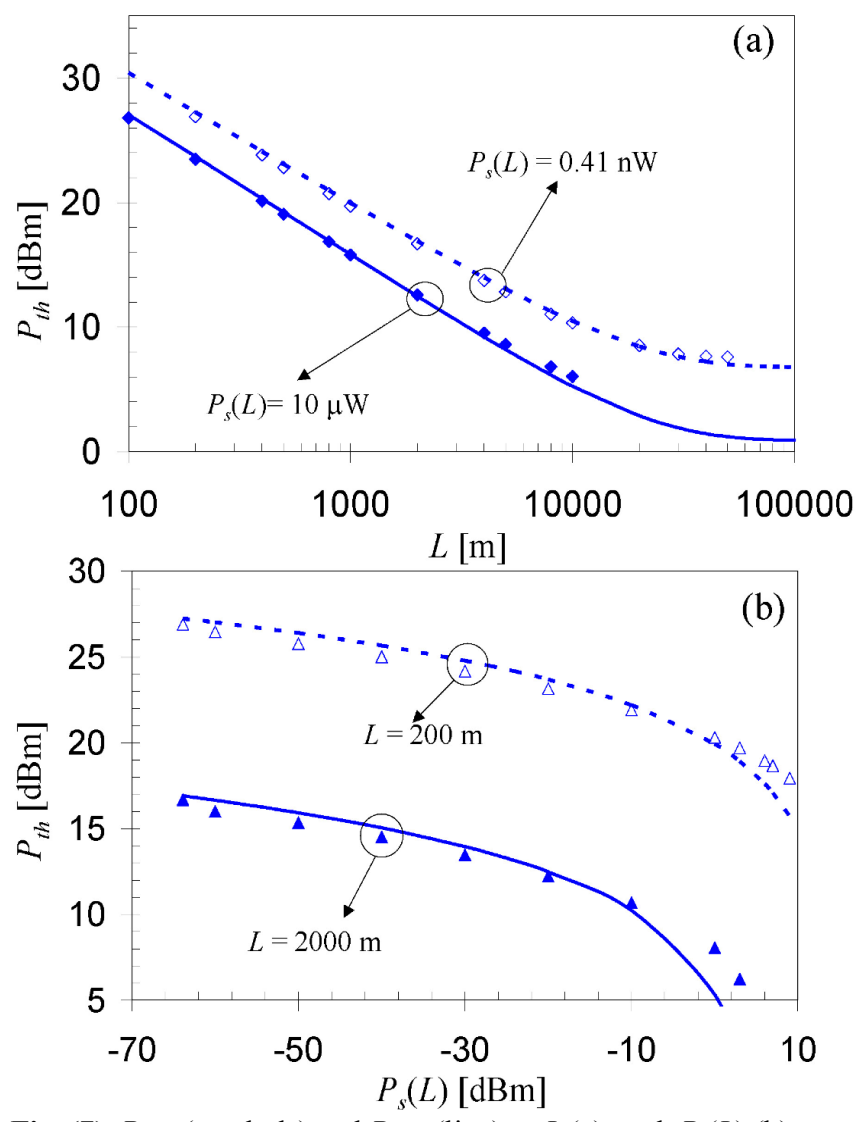

Fig. (7). $P_{\text {num }}$ (symbols) and $P_{B F A}$ (line) $v s L$ (a), and, $P_{s}(L)$ (b).

\section{CONCLUSION}

The inflexion method and the analytical relation (Eq. 6) have proven to be valid threshold definitions for the BFA configuration. Although the whole discussion was driven by considerations from the sensing field, we believe that these definitions have a broader range of applicability. In fact, many applications require the control of pump depletion as the Stokes spectrum suffers gain saturation and poor signalto-noise ratio at higher power $[15,16]$. The threshold power is the maximum power that could be used in the sensor system.

\section{ACKNOWLEDGEMENTS}

The authors would like to acknowledge the financial support of Intelligent Sensing for Innovative Structures Canada, Natural Science and Engineering Research Council Canada, the Canadian Foundation for Innovation and Agile All-Photonic Networks. The authors also would like to thank Aragon Photonics for kindly loaning the BOSA.

\section{REFERENCES}

[1] Olsson NA, van der Ziel JP. Cancellation of fiber loss by semiconductor laser pumped Brillouin amplification at $1.5 \mu \mathrm{m}$. Appl Phys Lett 1972; 48: 1329-30.

[2] Tkach RW, Chraplyvy AR, Derosier RM. Performance of a WDM network based on stimulated Brillouin scattering. IEEE Photo Tech Lett 1989; 1: 111-3. 
[3] Okawachi Y, Bigelow MS, Sharping JE, et al. Tunable all-optical delays via Brillouin Slow Light in an Optical Fiber. Phys Rev Lett 2005; 94: 153902-1-4.

[4] Song K-Y, González-Herráez M, Thévenaz L. Observation of pulse delaying and advancement in optical fibers using stimulated Brillouin scattering. Opt Express 2005; 13: 9758-65.

[5] Horiguchi T, Kurashima T, Tateda M. Tensile strain of Brillouin frequency shift in silica optical fibers. IEEE Photo Tech Lett 1989; 1: 107-9.

[6] Culverhouse D, Frahi F, Pannell CN, Jackson DA. Potential of stimulated Brillouin scattering as sensing mechanism for distributed temperature sensor. Electron Lett 1989; 25: 913-14.

[7] Ravet F, Chen L, Bao X, Zou L, Kalosha VP. Theoretical study of the effect of slow light on BOTDA spatial resolution. Opt Express 2006: 14: 10351-8.

[8] Smith RG. Optical power handling capacity of low loss optical fibers as determined by stimulated Raman and Brillouin scattering. Appl Optics 1972; 11:2489-94

[9] Van Deventer O, Boot AJ. Polarization Properties of Stimulated Brillouin scattering in single-mode fibers. J Ligthwave Technol 1994; 12: 585-90.
[10] Bayvel P, Radmore PM. Solutions of the SBS equations in singlemode optical fibers and implications for fiber transmission systems. Electron Lett 1990; 26: 434-5.

[11] Cotter D. Observation of stimulated Brillouin scattering in low-loss silica fiber at 1.3- $\mu \mathrm{m}$. Electron Lett 1982; 18: 495-6.

[12] Derickson D, Ed. Fiber optic tests and measurements. Prentice Hall: New Jersey; 1998.

[13] Villafranca A, Lázaro JA, Salinas I. Stimulated Brillouin scattering gain profile characterization by interaction between two narrowlinewidth optical sources. Opt Express 2005; 13: 7336-41.

[14] ITU-T G.652 2000. Series G: Transmission Systems and Media, Digital Systems and Networks. International Telecommunication Union.

[15] Boyd RW, Rzazewski K, Narum P. Noise initiation of stimulated Brillouin scattering. Phys Rev A 1990; 42: 5514-21.

[16] Ferreira MF, Rocha JF, Pinto JL. Analysis of the gain and noise characteristics of fiber Brillouin amplifiers. Opt Quantum Electron 1994; 26: 35-44

(C) Ravet et al.; Licensee Bentham Open.

This is an open access article licensed under the terms of the Creative Commons Attribution Non-Commercial License (http://creativecommons.org/licenses/by-nc/ $3.0 /$ ) which permits unrestricted, non-commercial use, distribution and reproduction in any medium, provided the work is properly cited. 